\title{
Non-Markovian Model for Transport and Reactions of Particles in Spiny Dendrites
}

\author{
Sergei Fedotov ${ }^{1}$ and Vicenç Méndez ${ }^{2}$ \\ ${ }^{1}$ School of Mathematics, The University of Manchester, Manchester M60 1QD, United Kingdom \\ ${ }^{2}$ Grup de Física Estadística, Universitat Autònoma de Barcelona, 08193 Bellaterra Spain
}

(Received 30 July 2008; published 21 November 2008)

\begin{abstract}
Motivated by the experiments [Santamaria et al., Neuron 52, 635 (2006)] that indicated the possibility of subdiffusive transport of molecules along dendrites of cerebellar Purkinje cells, we develop a mesoscopic model for transport and chemical reactions of particles in spiny dendrites. The communication between spines and a parent dendrite is described by a non-Markovian random process and, as a result, the overall movement of particles can be subdiffusive. A system of integrodifferential equations is derived for the particles densities in dendrites and spines. This system involves the spine-dendrite interaction term which describes the memory effects and nonlocality in space. We consider the impact of power-law waiting time distributions on the transport of biochemical signals and mechanism of the accumulation of plasticity-inducing signals inside spines.
\end{abstract}

PACS numbers: 87.19.L,- 05.40.- a, 05.60. $-\mathrm{k}$

Dendritic spines are essential elements of most brain regions because they form a surface for receiving synaptic inputs. For the Purkinje cells of the cerebellar cortex, over $90 \%$ of their excitatory synapses are located on dendritic spines. They are believed to play a very important role in regulating neuronal activity [1-3]. The heads of spines have an active membrane; therefore, the spiny dendrites can sustain the propagation of an action potential. The propagation rate depends on the spatial distribution of spines along dendrites. Much theoretical work has been devoted to studying the interaction of spines with dendrites on the macroscopic level. Baer and Rinzel [4] proposed a cable theory for excitable spiny dendrites and found that the spread of local excitation strongly depends on a spinestem resistance. There are many extensions of this work that take into account the dynamic structure of spines and their changes in response to synaptic activity [5]. Coombes and Bressloff suggested the simplified version of classical theory based on FitzHugh-Nagumo model [6]. Note that these cable models are phenomenological and not derived from the transport and biochemical reactions equations for ions in spiny dendrites. In recent years, the development of confocal microscopies and other techniques allow one to study the transport and biochemical reactions on the microscopic level of a single spine and a parent dendrite [7-9]. There are several models for the particle transport and chemical reactions inside biological microdomains [1013]. Santamaria et al. [14] found recently that the transport of biologically inert particles (fluorescein dextran) in spiny dendrites is very slow in comparison to the standard diffusion. The mean-square displacement is $\left\langle x^{2}(t)\right\rangle \sim t^{\mu}$ with $\mu<1[15,16]$. The authors suggested that the main reason for this anomalous diffusion is that the dendritic spines act as the traps of particles. Henry et al. [17] addressed this problem by proposing a cable model derived from fractional Nernst-Planck equations. Whereas many studies have been devoted to the coupling between spines and den- drites, they are either phenomenological cable theories or microscopic models for a single spine and parent dendrite. To the authors knowledge, there are still no mesoscopic models for the transport and biochemical reactions inside the population of spines and dendrites. We stress that abnormalities in dendritic spine populations (e.g., decreased spine density) may result in cognitive disorders such as autism, mental retardation, and fragile $X$ syndrome [2].

The purpose of this Letter is to set up a mesoscopic model for the transport of particles inside a single spiny dendrite and chemical reaction in spines. Morphology of spiny dendrites is very complex: the distances between the spines, their sizes, and their shapes are randomly distributed $[1,18]$. Our model allows us to deal with morphological diversity of dendritic spines through the transparent formalism of waiting time distributions. We adopt the following stochastic model. Inside a dendrite with the uniform surface spine density $\rho_{s}$, a particle performs a Brownian motion with constant drift $v$ along dendrite and a diffusion coefficient $D$. This particle can describe movement of an overdamped ion under the action of an electrical field $E$ such that $v=\mu E$, where $\mu$ is the ion mobility. After a random time $\tau_{d}$ distributed by the probability density function (PDF) $\psi_{1}(\tau)$ the particle hits the neck of spine on the surface of dendrite. Then it is trapped inside the spine for a random time $\tau_{s}$. The PDF for the dwelling time $\tau_{s}$ is $\psi_{2}(\tau)$. During this time irreversible chemical reaction $C \stackrel{\beta}{\rightarrow} C_{b}$ takes place, where $\beta$ is the rate of removing particles by buffers and pumps inside spines. After the random time $\tau_{s}$, the particle is released to the parent dendrite through spine neck again. The experimental evidence indicates that spine necks act as a transport barrier. The measurements of the $\mathrm{Ca}^{2+}$ flux out of spines show that spine necks slow down the transport of ions up to a factor $10^{2}$ compared with free diffusion [8]. For these reasons we suggest a power-law distribution for the dwelling time PDF $\psi_{2}(\tau)$, which also accounts for heteroge- 
neous spine-dendrite transport coupling [9]. Note that calcium dynamics inside spines is a very complex process regulated by endogenous $\mathrm{Ca}^{2+}$ buffers, $\mathrm{IP}_{3}$-mediated calcium release from intracellular stores, pumps on the spine membrane, etc. [8]. These factors also contribute to the effective dwelling time $\tau_{s}$. The PDF $\psi_{1}(\tau)$ for the random time $\tau_{d}$ can be found from the escape problem for a diffusion in bounded domain with absorbing sites (spines) on the surface [10]. If a cylindrical dendrite of length $L$ and radius $R$ has just one spine with neck radius $a \ll R$, then the survival probability $\int_{t}^{\infty} \psi_{1}(s) d s$ is exponentially distributed for large $t$ and the mean escape time $\left\langle\tau_{d}\right\rangle$ is $\pi R^{2} L / 4 a D[10,13]$. In this Letter we use two distributions: exponential $\quad \psi_{1}(t)=\gamma_{1} \exp \left(-\gamma_{1} t\right) \quad$ (Markovian) and gamma distribution $\psi_{1}(t)=\nu_{1}^{2} t e^{-\nu_{1} t}$ (non-Markovian). In both cases it is assumed that the parameters $\gamma_{1}$ and $\nu_{1}$ are functions of the linear spine density $\rho_{l}=2 \pi R \rho_{s}$, that is $\gamma_{1}=\rho_{l} \gamma_{1}^{0}$ and $\nu_{1}=\rho_{l} \nu_{1}^{0}$. Its typical value for dendrites with radius $0.5 \mu \mathrm{m}$ is around 10 spines per $\mu \mathrm{m}$ [14]. Because of rather general character of our model, it is not confined to the spiny dendrites, but it also allows us to address the general issues of the transport and reactions in fractals, porous media and disordered systems such as nanochannel materials [19], biofilms [20], etc.

The main quantities of interest in this Letter are the density of particles, $n_{1}=n_{1}(x, t)$, inside a single dendrite and the density of particles, $n_{2}=n_{2}(x, t)$ inside a single spine, where $x$ is the distance along a dendrite. Let us derive the balance equations for $n_{1}$ and $n_{2}$ by using the stochastic model for a single particle proposed above. Let $n_{1}^{0}$ and $n_{2}^{0}$ be the initial densities of particles in dendrite and spine. If $j_{1}(x, t)$ denotes the number of particles arriving at point $x$ in a dendrite at time $t$ through a single spine stem and $j_{2}(x, t)$ is the number of particles arriving at point $x$ in a single spine at time $t$ (see Fig. 1), the balance equation for the densities $n_{1}$ and $n_{2}$ can be written as follows:

For a dendrite

$$
n_{1}(x, t)=\Psi_{1}(t) \mathbf{E}_{x, t}\left[n_{1}^{0}\right]+\rho_{l} \int_{0}^{t} \Psi_{1}(u) \mathbf{E}_{x, t-u}\left[j_{1}\right] d u .
$$

For a single spine

$$
n_{2}(x, t)=\Psi_{2}(t) e^{-\beta t} n_{2}^{0}(x)+\int_{0}^{t} \Psi_{2}(u) e^{-\beta u} j_{2}(x, t-u) d u .
$$

Here we introduce the expectation operator $\mathbf{E}_{x, t}$ :

$$
\mathbf{E}_{x, t}\left[n_{1}^{0}\right]=\int_{-\infty}^{\infty} p(x, t \mid z) n_{1}^{0}(z) d z,
$$

where $p(x, t \mid z)$ is the probability density function for a particle which starts at point $z$ in a dendrite and ends up at point $x$ at time $t$. Equation (1) gives the density of particles at point $x$ in a dendrite at time $t$ as a sum of two terms. The first term $\Psi_{1}(t) \mathbf{E}_{x, t}\left[n_{1}^{0}\right]$ is the contribution of those particles that are initially located inside the dendrite and diffuse along it from $t=0$ up to time $t$ without moving into spines. The second term represents those particles that arrived in the dendrite from spines at point $z$ at time $t-u$

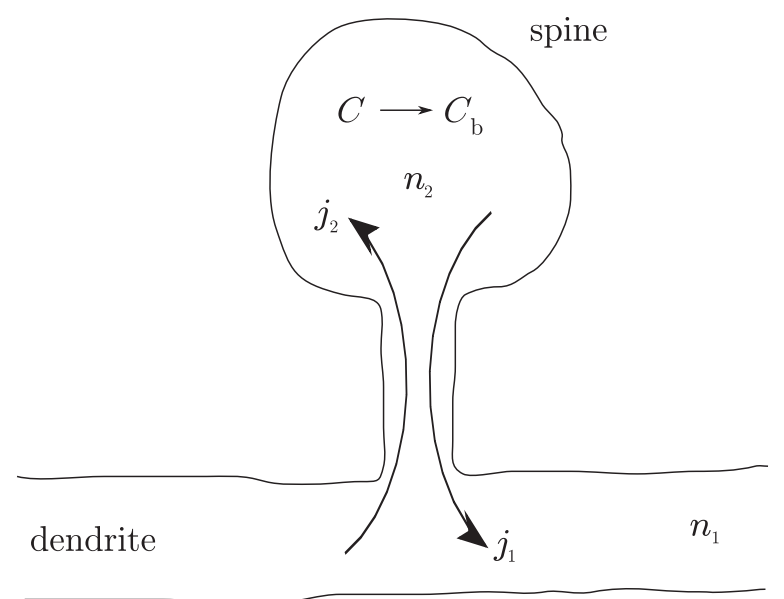

FIG. 1. Spine-dendrite interaction.

and diffuse to reach point $x$ without switching to spines. The functions $\Psi_{1}(t)$ and $\Psi_{2}(t)\left[\Psi_{i}(t)=\int_{t}^{\infty} \psi_{i}(u) d u, i=\right.$ $1,2]$ are the survival probabilities of particles in dendrites and spines, respectively. Equation (2) is the particle's balance equation in a single spine. Note that $\mathbf{E}_{x, t-u}\left[j_{1}\right]=$ $\int_{-\infty}^{\infty} p(x, t-u \mid z) j_{1}(z, t-u) d z$. It gives the density of free particles that are not bounded to absorbing sites inside spine. The function $e^{-\beta t}$ gives the fraction of free particles. Then the first term $\Psi_{2}(t) e^{-\beta t} n_{2}^{0}(x)$ is the contribution from particles initially located in a spine that remain free up to time $t$ without moving inside dendrite. Now let us formulate the balance equations for $j_{1}(x, t)$ and $j_{2}(x, t)$.

For a dendrite

$j_{1}(x, t)=\psi_{2}(t) e^{-\beta t} n_{2}^{0}(x)+\int_{0}^{t} \psi_{2}(u) e^{-\beta u} j_{2}(x, t-u) d u$.

For a single spine

$$
j_{2}(x, t)=\psi_{1}(t) \mathbf{E}_{x, t}\left[n_{1}^{0}\right] / \rho_{l}+\int_{0}^{t} \psi_{1}(u) \mathbf{E}_{x, t-u}\left[j_{1}\right] d u .
$$

The first term in (4) is the number of particles initially located in spine that arrive at dendrite at time $t$ due to the transport through spine neck. The second term represents those particles that arrived at spines at point $x$ at time $t-u$, spent time $u$ before moving into dendrite at time $t$. In (5) the same balance of particles is applied, but we take into account the motion of particles along the dendrite. Note that all balance equations are valid in a nonlinear case when the electric field $E$ is induced by a membrane potential which depends on ions concentrations. This can be included into the PDF $p$. In what follows we consider $v=$ const. For this linear problem, one can obtain an explicit expression for PDF

$$
p(x, t \mid z)=(4 \pi D t)^{-1 / 2} \exp \left[-(x-z-v t)^{2} / 4 D t\right] .
$$

By using the Fourier-Laplace transforms $(x, t) \rightarrow(q, s)$, one can find from the balance Eqs. (1)-(5) the expressions for $s n_{i}(q, s)-n_{i}^{0}(q)$. By using the Fourier-Laplace inver- 
sion formula, we obtain a system of integrodifferential equations

$$
\begin{gathered}
\frac{\partial n_{1}}{\partial t}+v \frac{\partial n_{1}}{\partial x}=D \frac{\partial^{2} n_{1}}{\partial x^{2}}-\rho_{l} J\left[n_{1}, n_{2}\right], \\
\frac{\partial n_{2}}{\partial t}=J\left[n_{1}, n_{2}\right]-\beta n_{2},
\end{gathered}
$$

with the spine-dendrite interaction term

$$
\begin{aligned}
J\left[n_{1}, n_{2}\right]= & -\int_{0}^{t} e^{-\beta u} a_{2}(u) n_{2}(x, t-u) d u \\
& +\rho_{l}^{-1} \int_{-\infty}^{\infty} \int_{0}^{t} p(x, u \mid z) a_{1}(u) n_{1}(x-z, t \\
& -u) d u d z,
\end{aligned}
$$

where the Laplace transforms of kernels are $\tilde{a}_{i}(s)=$ $\tilde{\psi}_{i}(s) / \tilde{\Psi}_{i}(s), \quad i=1,2$. The symbol $\tilde{f}$ indicates the Laplace transform of $f$. Note that our model involves two equations for freely moving particles and particles trapped in the spines whereas the standard CTRW model describes the trapping property as a temporal memory in the moving particles. The derivation of nonstandard spinedendrite interaction term (9) is one of the main result of this Letter. $J\left[n_{1}, n_{2}\right]$ is the number of particles per unit time flowing between the dendrite and a single spine. The first term in the right-hand side of (9) describes the flux of particles from a single spine through spine neck into a parent dendrite and the second term gives the flux of particles from dendrite into spine. The spines-dendrite coupling is crucial for the propagation of action potential along spiny dendrites since there is no direct communication between neighboring spines. It turns out that this interaction is far from trivial and cannot be easily found by using phenomenological approach. In general both terms in are nonlocal in time. One can see from (9) that the effective memory kernel in the first term depends on chemical reaction in spines with decay rate $\beta$. The second term of (9) is nonlocal in space. Note that this effect is not due to the long-range jumps of particles inside the dendrite. This nonlocal behavior may lead to anomalous transport of particles in dendrites (see below). The main conclusion is that there is no separation of transport and chemical reactions from interaction between spines and dendrite in general case (see similar phenomenon in [21-24]). It happens only in a simple Markovian case. If the probability density functions $\psi_{1}(t)$ and $\psi_{2}(t)$ are exponential with the rates $\gamma_{1}=\left\langle\tau_{d}\right\rangle^{-1}$ and $\gamma_{2}=\left\langle\tau_{s}\right\rangle^{-1}$, then the memory kernels $a_{i}(t)$ are delta functions and the system (7) and (8) takes the form of standard reaction-diffusion equations with the interaction term

$$
J\left[n_{1}, n_{2}\right]=\gamma_{1}^{0} n_{1}-\gamma_{2} n_{2},
$$

where $\gamma_{1}^{0}=\gamma_{1} / \rho_{l}$. This Markovian model corresponds to the phenomenological approach for spine-dendrite interaction [12]. Consider now the non-Markovian case when both waiting time PDFs $\psi_{i}$ are gamma distributions $\psi_{i}(t)=\nu_{i}^{2} t e^{-\nu_{i} t}, i=1,2$. Then the interaction memory kernels are $a_{i}(t)=\nu_{i}^{2} e^{-2 \nu_{i} t}, i=1,2$. This formula shows that the effective memory kernel in the first term is $e^{-\left(2 \nu_{1}+\beta\right) t}$. The effective delay time is $\left(2 \nu_{1}+\beta\right)^{-1}$. When the drift is zero, the second term can be written in the Fourier space as $\nu_{1}^{2} \int_{0}^{t} e^{-\left(2 \nu_{1}+D q^{2}\right) u} n_{1}(q, t-u) d u$. It means that the effective delay time $\left(2 \nu_{1}+D q^{2}\right)^{-1}$ depends on the diffusion in dendrites and the density of spines since $\nu_{1}=\rho_{l} \nu_{1}^{0}$.

Biologically inert particles: anomalous diffusion.-It has been found recently that the transport of inert particles (fluorescein dextran) in spiny dendrites of cerebellar Purkinje cells is subdiffusive [14], i.e., $\left\langle x^{2}(t)\right\rangle \sim t^{\mu}$, where $0<\mu<1$. Let us show that our model also predicts this subdiffusive behavior. Consider the case when there is no binding reaction inside the spines: $\beta=0$. For simplicity we assume that waiting time PDF for dendrite $\psi_{1}(t)$ is exponential. On the basis of the experimental evidence, we suggest a power-law distribution for the waiting time density $\psi_{2}(t) \sim(t / \tau)^{-1-\mu}$ as $t \rightarrow \infty$, which in the Laplace space is $\tilde{\psi}_{2}(s)=\left(1+(\tau s)^{\mu}\right)^{-1}[15,16]$. The Laplace transform of the mean squared displacement is $\left\langle x^{2}(s)\right\rangle=$ $-\frac{d^{2} n}{d q^{2}}(0, s)$; here $n(q, s)=n_{1}(q, s)+\rho_{l} n_{2}(q, s)$ is the Laplace-Fourier transform of the total density of particles. One can obtain from (1)-(5)

$$
\begin{aligned}
n(q, s)= & n_{1}^{0}(q) \frac{\tilde{\Psi}_{1}^{q}(s)+\tilde{\Psi}_{2}(s+\beta) \tilde{\psi}_{1}^{q}(s)}{1-\tilde{\psi}_{2}(s+\beta) \tilde{\psi}_{1}^{q}(s)} \\
& +\rho_{l} n_{2}^{0}(q) \frac{\tilde{\Psi}_{2}(s+\beta)+\tilde{\psi}_{2}(s+\beta) \tilde{\Psi}_{1}^{q}(s)}{1-\tilde{\psi}_{2}(s+\beta) \tilde{\psi}_{1}^{q}(s)},
\end{aligned}
$$

where $\tilde{\Psi}_{1}^{q}(s)=\tilde{\Psi}_{1}\left(s+i q v+D q^{2}\right)$. In particular, when $\beta=0$ and $v=0$, we compute the mean squared displacement

$$
\left\langle x^{2}(t)\right\rangle \simeq\left\langle\tau_{d}\right\rangle D(t / \tau)^{\mu}
$$

as $t \rightarrow \infty$. In the Markovian case with PDFs $\psi_{i}(t)=$ $\gamma_{i} \exp \left(-\gamma_{i} t\right)$, one can find the standard $\left\langle x^{2}(t)\right\rangle \simeq D_{\text {eq }} t$, where the effective diffusivity $D_{\text {eq }}=D \gamma_{2}\left(\gamma_{1}+\gamma_{2}\right)^{-1}$. In the anomalous case (12) the effective diffusion coefficient tends to zero. Note that the authors in [14] measured the mean-square displacement (MSD) as ensemble average. It has been found recently [25] that the time averaged MSD is different from ensemble averaged MSD for subdiffusion. This is a clear indication of ergodicity breaking which is closely related to the aging property of the CTRW with power-law waiting times.

Kinetics of particles decay in spiny dendrites.-While anomalous switching slows down the transport of particles in dendrites (12), it leads to a high rate of particles' decay compared to the Markovian case. This is illustrated in Fig. 2 where we compared the decay of particles in an anomalous case $(\mu=0.1)$ with that in the non-Markovian case (gamma distribution) and standard Markovian case $(\mu=1)$. One can see that the decrease of diffusion of particles towards the parent dendrite (decrease of the ex- 


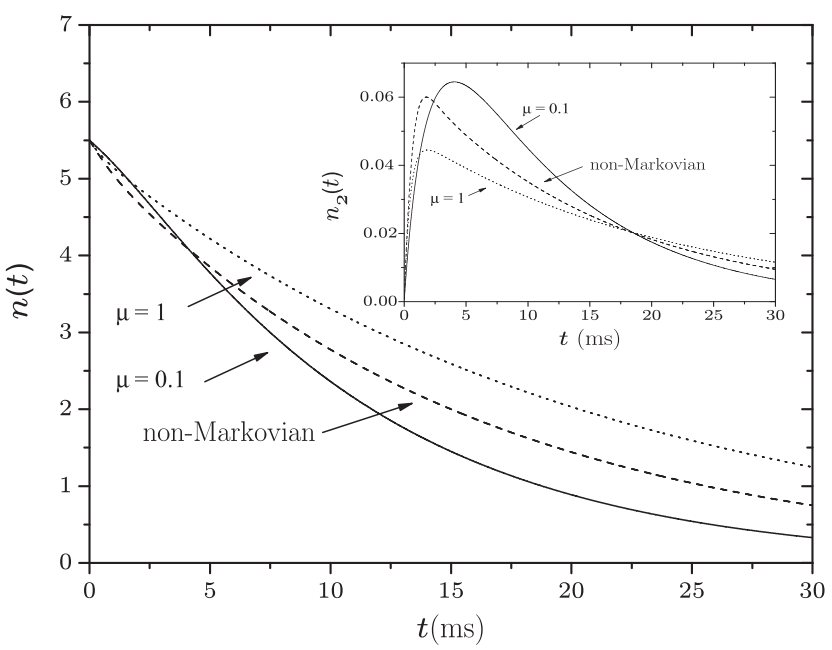

FIG. 2. Kinetics of particles decaying in the system spinedendrite $n(t)$ (main figure) and in spines $n_{2}(t)$ (inset). $D=0.2$, $\mu \mathrm{m}^{2} / \mathrm{ms},\left\langle\tau_{s}\right\rangle=\left\langle\tau_{d}\right\rangle=1 \mathrm{~ms}, \beta=0.1 \mathrm{~ms}^{-1}, \rho_{l}=10 \mu \mathrm{m}^{-1}$. The initial conditions are $n_{i}(x, 0)=\delta(x) / 2, i=1,2$.

ponent $\mu$ ) leads to a faster decay of total number of particles $n(t)=\int\left(n_{1}+\rho_{l} n_{2}\right) d x$. From Eq. (12), $n(t) \sim$ $t^{-\mu} e^{-\beta t}$ as $t \rightarrow \infty$. This explains the effect of limited diffusion of $\mathrm{Ca}^{2+}$ along dendrites observed in experiments [14]. The decay of $n_{2}$ is also illustrated in Fig. 2 showing that transport equilibration is slow. This is very important for the accumulation of plasticity-inducing signals inside spines [9]. The phenomenological model for spinedendrite interaction assumes that the ion current $I$ passing through the spine necks is proportional to the current voltage difference between the spines and dendrite. Our findings demonstrate that particles flux through the spine's neck is nonlocal in time and space [see (9)]. In general, the ion current $I\left[\varphi_{1}, \varphi_{2}\right]=\iint_{0}^{t} G(z, u)\left(\varphi_{1}-\varphi_{2}\right) d u d z$, where the kernel $G(z, u)$ has to be determined. Note that Henry et al. [17] used the spine-dendrite ion current with the Riemann-Liouville fractional derivative operator instead of local Ohm's law.

In summary, we have developed the mesoscopic model for transport and reactions of particles inside spiny dendrites. The main feature of our model is that the spinedendrite interaction has been described by a nonMarkovian random process giving rise to a flux of particles between the dendrite and spines that is nonlocal in space and time (memory effects). This allows us to explain two important effects observed in experiments [14]: (i) a subdiffusive transport of inert molecules along spiny dendrites and (ii) limited diffusion of a second messenger $\left(\mathrm{Ca}^{2+}\right)$ due to fast effective decay of particles inside spines. Because of the non-Markovian character of spine-dendrite interaction, a significant delay of diffusional equilibration between spines and dendrites takes place. As a result, the local rises in concentration of biochemical signals generated by synaptic activation may lead to accumulation of plasticityinducing signals inside spines [9]. This is important for understanding the mechanisms of synaptic plasticity which underlie learning and memory.

We acknowledge a fruitful discussion with Susan Wearne. This research has been partially supported by the Generalitat de Catalunya with the Grants No. SGR 2005-00087 (V. M.), No. 2007-PIV-00001 (S. F.), and by Grant No. FIS 2006-12296-C02-01 (V.M.). The authors thank the Institute for Mathematics and its Applications (IMA), of the University of Minnesota for financial support and great hospitality.

[1] K. M. Harris, Curr. Opin. Neurobiol. 9, 343 (1999).

[2] E. Nimchinsky, B. Sabatini, and K. Svoboda, Annu. Rev. Physiol. 64, 313 (2002).

[3] M. Segal, Nat. Rev. Neurosci. 6, 277 (2005).

[4] S. M. Baer and J. Rinzel, J. Neurophysiol. 65, 874 (1991).

[5] H-Y. Wu and S. M. Baer, J. Math. Biol. 36, 569 (1998).

[6] S. Coombes and P. C. Bressloff, SIAM J. Appl. Math. 61, 432 (2000); Phys. Rev. Lett. 91, 028102 (2003).

[7] K. Svoboda, D. W. Tank, and W. Denk, Science 272, 716 (1996).

[8] B. L. Sabatini, M. Maravall, and K. Svoboda, Curr. Opin. Neurobiol. 11, 349 (2001).

[9] B.L. Bloodgood and B.L. Sabatini, Science 310, 866 (2005).

[10] Z. Schuss, A. Singer, and D. Holcman, Proc. Natl. Acad. Sci. U.S.A. 104, 16098 (2007).

[11] D. Holcman, A. Marchewka, and Z. Schuss, Phys. Rev. E 72, 031910 (2005); D. Holcman and Z. Schuss, SIAM J. Appl. Math. 65 , 1006 (2005).

[12] P.C. Bressloff and B. A. Earnshaw, Phys. Rev. E 75, 041915 (2007).

[13] L. Dagdug, A. M. Berezhkovskii, Yu. A. Makhnovskii, and V. Yu. Zitserman, J. Chem. Phys. 127, 224712 (2007).

[14] F. Santamaria, S. Wils, E. De Schutter, and G. J. Augustine, Neuron 52, 635 (2006).

[15] R. Metzler and J. Klafter, Phys. Rep. 339, 1 (2000).

[16] M. Saxton, Biophys. J. 70, 1250 (1996).

[17] B. I. Henry, T. A. M. Langlands, and S. L. Wearne, Phys. Rev. Lett. 100, 128103 (2008).

[18] A. Rodriguez, D. B. Ehlenberger, D. L. Dickstein, P. R. Hof, and S. L. Wearne, PLoS ONE 3, 1 (2008)

[19] A. Berezhkovskii and G. Hummer, Phys. Rev. Lett. 89, 064503 (2002).

[20] J. D. Seymour, J. P. Gage, S. L. Codd, and R. Gerlach, Phys. Rev. Lett. 93, 198103 (2004).

[21] A. Yadav and W. Horsthemke, Phys. Rev. E 74, 066118 (2006).

[22] S. Fedotov and A. Iomin, Phys. Rev. Lett. 98, 118101 (2007); Phys. Rev. E 77, 031911 (2008).

[23] D. Froemberg and I. M. Sokolov, Phys. Rev. Lett. 100, 108304 (2008); F. Sagués, V. P. Shkilev, I. M. Sokolov, and M. G. W. Schmidt, Phys. Rev. E 77, 032102 (2008).

[24] B. I. Henry, T. A. M. Langlands, and S. L. Wearne, Phys. Rev. E 74, 031116 (2006).

[25] Y. He, S. Burov, R. Metzler, and E. Barkai, Phys. Rev. Lett. 101, 058101 (2008). 